\title{
MODERNISMO GLOBAL Y LITERATURA MUNDIAL: REFLEXIONES SOBRE LAS DISLOCACIONES COSMOPOLITAS DEL SIGNIFICANTE FRANCÉS ${ }^{1}$
}

\author{
Mariano Siskind \\ Harvard University, Cambridge, Estados Unidos \\ siskind@fas.harvard.edu
}

RESUMEN / ABSTRACT

\begin{abstract}
Este artículo propone una aproximación contingente y descentrada del mundo como fundamento para pensar una literatura mundial. Describe, en ese marco, la construcción de un significante francés por parte de los modernistas latinoamericanos, un París universal respecto del cual podrán constituirse como particularidad marginal. En oposición a ese artefacto, se discute el imaginario mundial de la novela experimental L'Homme de la pampa (1923), del escritor franco-uruguayo Jules Supervielle, cuya adscripción identitaria es doblemente intersticial (nunca asimilado al surrealismo francés, mantenido a distancia en el Uruguay). El mundo de Supervielle es un mundo dislocado y heterogéneo, sin formaciones estables y, por tanto, resistente a la fetichización de la diferencia cultural, incluida la que se construye alrededor de la diferencia latinoamericana.
\end{abstract}

PALABRAS CLAVE: literatura mundial, literaturas del mundo, francofilia, intersticio, Jules Supervielle.

1 Una versión diferente de este artículo fue publicada en inglés bajo el título "Dislocating France. World Literature, Global Modernism and Cosmopolitan Distance" en el Journal of World Literature. Special Issue: Rethinking World Literature Studies in Latin American and Spanish Contexts 2.1 (2017): 47-62. Quiero agradecer a Rodrigo del Río Joglar por su ayuda con la traducción. 
Global Modernism and World Literature: Thoughts on the Cosmopolitan Dislocations of THE FRENCH SIGNIFIER

This article proposes a dis-centered and contingent approach to the world as basis to think about world literature. In this frame, the construction of a French significant by Latin American modernists is described, a universal Paris from which they will articulate themselves as a marginal particularity. In opposition to such artefact, the article discusses the world imaginary in the experimental novel L'Homme de la pampa (1923), by French-Uruguayan writer Jules Supervielle, whose identity adscription is doubly interstitial: never assimilated to French surrealism and kept at distance in Uruguay. Supervielle's world is dislocated and heterogeneous, without stable formations and, therefore, resistant to the fetishization of cultural difference, including that which is built upon Latin American difference.

KEYWORDS: world literature, literatures of the world, francophilia, interstice, Jules Supervielle. Recepción: 31/07/2017

Aprobación: 18/08/2017

\section{EL MUNDO NO EXISTE}

La idea de la inexistencia del mundo, o bien, de que el mundo no preexiste a sus articulaciones contingentes en nuestros discursos críticos es el punto de partida ineludible para una aproximación crítica antifundacionalista del mundo, es decir, del mundo tal como fue entendido en el contexto del discurso de la modernidad: una totalidad imaginaria sobre la que se despliega, con potencias y limitaciones evidentes, el discurso crítico de la literatura mundial y del modernismo global. Esta afirmación, el mundo no existe, entonces, desestabiliza la mundialidad de la literatura mundial y la globalidad del modernismo global porque supone la imposibilidad de dar por sentada la estructura simbólica y material que funciona como base para cualquier invocación más o menos acrítica y preteórica de nociones como circulación global y traducción transcultural (y cualquiera de los predicados que se suelen usar, mundial, global, transnacional) sobre los que se erigió el discurso crítico de la literatura mundial, de Goethe a David Damrosch. El punto es que el mundo no es, ni debe ser considerado, un datum; el mundo no es (no puede ser) lo dado; no es (ni puede ser) un supuesto que preexista a las intervenciones críticas o estéticas que tienen la obligación de postular en términos contingentes y concretos, mundo o no-mundo, la estructura que sostiene y soporta su intervención discursiva. Todo texto que trasciende la inmediatez de lo local postula un mundo, una formación transcultural distinta 
y singular que podemos (o no) llamar "mundo", dependiendo de si queremos o no crear la ilusión falaz en nuestra audiencia de que el "mundo" (entre comillas para referir a la estructura contingente sobre la que inscribimos nuestro discurso crítico) coincide con el mundo, con la totalidad imaginaria que proyectamos sobre el planeta.

Tomo prestada la formulación de esta idea del mundo como la estructura simbólica que no preexiste el momento de su postulación discursiva del libro fundacional Hegemonía y estrategia socialista, de Ernesto Laclau y Chantal Mouffe, en el que ellos se apropian y resignifican el famoso dictum de Margaret Thatcher según el cual "la sociedad no existe". Si Thatcher decía esto como negación neoliberal de cualquier tipo de colectividad, y proponía, en cambio, que la única existencia constatable y positiva era la de individuos aislados cuya ciudadanía estaba determinada por su condición de propietarios, consumidores y contribuyentes impositivos (una concepción de lo social que pervive hoy en el reclamo del achicamiento del estado al grito de "¡esto lo pago yo con mis impuestos!"), Laclau y Mouffe resignifican y se apropian de la idea de que "la sociedad no existe" como crítica postmarxista de cualquier forma de objetividad positiva, particularmente de la supuesta objetividad de la clase social como estructura socio-económica que sobredetermina la distribución de las identidades proletarias y burguesas a partir de las que se organizan las relaciones de producción modernas. Es decir, para Laclau y Mouffe la sociedad no existe como el espacio estructural de significación, ya que los procesos de subjetivación y significación son para ellos siempre el resultado de articulaciones hegemónicas contingentes. En función de esto, voy a citar un pasaje de Hegemonía y estrategia socialista, y espero que, cuando los autores refieren a "lo social", "la sociedad" y "totalidad", los lectores los reemplacen imaginariamente por "el mundo" y "lo mundial" o "lo global":

Consideramos lo social como espacio no suturado, como campo en que toda positividad es metafórica y subvertible. . El antagonismo como negación de un cierto orden es, simplemente, el límite de dicho orden y no el momento de una totalidad más amplia respecto a la cual los dos polos del antagonismo constituirían instancias diferenciales -es decir, objetivas- parciales. . . El límite de lo social debe darse en el interior mismo de lo social como algo que lo subvierte, es decir, como algo que destruye su aspiración a constituir una presencia plena. La sociedad no llega a ser totalmente sociedad porque todo en ella está penetrado por sus límites que le impiden constituirse como realidad objetiva (146-7). 
Esta caracterización de lo social puede ser traducida a una concepción del mundo -el mundo de la literatura mundial y del modernismo global en el contexto del argumento que intento proponer aquí- como un espacio no suturado y no suturable, constituido por antagonismos modernistas, deseos y voluntades estéticas en conflicto, y exclusiones institucionales que impiden la constitución de este mundo modernista como una totalidad de textos literarios idéntica a sí misma, cuya comparabilidad dependería del hecho de que circulan en torno de la misma estructura mundial (así, en cursivas o entre comillas). Lo que quiero decir es que, obviamente, la sutura del mundo es una fantasía, que el mundo como totalidad de sentido como marco para la conceptualización e institucionalización de relaciones, traducciones, influencias, préstamos y reescrituras literarias es una ficción hegemónica que el discurso crítico puede desestabilizar, dislocar y reconfigurar. Y en este sentido, lo que quiero proponer en este trabajo es que el mundo de la literatura mundial y el modernismo global no es, o no es únicamente, o no es principalmente, un mundo de conexiones, diálogos, colaboraciones, influencias y préstamos, un mundo donde los modernistas se citan y reescriben los unos a los otros; el mundo, por lo tanto, no como el sitio de encuentros modernistas experimentales y deseantes más allá de las asfixiantes determinaciones de lo local, lo nacional y lo regional. Este no es el mundo de la literatura mundial y el modernismo global, o al menos no es el mundo de la literatura mundial que a mí me interesa; no es el mundo de $m i$ literatura mundial ${ }^{2}$. De los múltiples mundos superpuestos y palimpsésticos que constituyen la estructura simbólica de la literatura mundial, mi mayor interés se cifra en los mundos habitados por cosmopolitas marginales, marcados por el deseo y el descontento, ansiosos de intervenir en esos mundos, listos para subvertir los órdenes que, según ellos, los marginan; mundos estructurados alrededor de tensiones irresueltas e irresolubles, desigualdad, antagonismos y exclusiones. Y en la medida en que sea un mundo de conexiones, encuentros y colaboraciones, estas redes de relaciones están determinadas por tensiones, desigualdades y exclusiones que dislocan el mundo, o más bien, que dislocan la estructura simbólica mundial/global que soporta los mundos de esos encuentros excluyentes, esas conexiones antagónicas que alimentan las estéticas modernistas desde

2 Esta es una alusión al momento en el que David Damrosch admite, en las conclusiones de su (re)fundacional libro What is world literature?, que "I have given you my world literature, or at least a representative cross-section of it" (281). 
siempre, en todas partes. Creo que siempre es así, incluso para cosmopolitas escribiendo desde lugares de enunciación hegemónicos, pero nunca es más manifiesto que en el caso de artistas y campos literarios marginales (y por marginales, me refiero tanto a la experiencia de exclusión de un artista del orden global de la modernidad, como a sitios geoculturales concretos).

Por ejemplo, los escritores latinoamericanos, y los modernistas marginales en general, imaginaron un mundo en el que los antagonismos que lo constituían estaban marcados por su autorrepresentación como escritores excluidos, por el deseo de inscribirse a sí mismos en los circuitos y redes del modernismo global, que ellos identificaban con el horizonte de significación que determinaba y autorizaba o no sus propias prácticas estéticas modernistas. En este sentido, Francia, París o lo que llamaré aquí el significante francés fue crucial para que estos escritores imaginaran mundos contingentes definidos por su radical diferencia y exterioridad respecto de su propia particularidad cultural; construyeron una Francia, un idioma francés y un París (el sitio mundial e histórico de las imaginaciones espaciales modernistas) como significantes inmediatamente instrumentalizables con la finalidad estratégica de inscribir su particularidad estética marginal en la universalidad imaginada del modernismo europeo (o una versión afrancesada del modernismo de Europa occidental). En efecto, citando a Lacan, lo francés sería un point de capiton alrededor del que se estructuró la totalidad del orden global de significación modernista.

Este ensayo es un intento de releer y reconceptualizar lo que usualmente se entiende de manera reduccionista como la francofilia de las literaturas marginales, y del modernismo latinoamericano (entendido en un sentido amplio) en particular. Si bien es evidente que en las élites culturales y políticas de las naciones marginales existe una pulsión francófila fuerte y verificable, lo que yo quiero decir es que en la relación con el significante francés hay mucho más que aquello que la tradición crítica regional/nacional popular llama peyorativamente "francofilia". En este sentido, este artículo no es sobre la creación francófila de los mundos imaginados por los modernistas marginales, ni sobre las reacciones y resistencias particularistas, antimiméticas y anticoloniales en su contra. Es más bien sobre la tensión entre el deseo por el significante francés de los modernistas marginales y sus intentos de desplazarlo y dislocarlo. Sobre cómo estos mundos marcados por la dislocación abren nuevos horizontes interpretativos para conceptualizar la literatura mundial y el modernismo global, no como intento disciplinario de los estudios literarios de afirmar un mundo más allá de las fronteras de la nación y la región, sino como discurso crítico y estético que busca dislocar el mundo, socavarlo, poner en duda su existencia o, mejor, su preexistencia; literatura mundial y modernismo global como formas de leer que subvierten y reconfiguran 
el orden del mundo, o mejor dicho, el orden de significación que estructura mundos de modo jerárquico, de menor a mayor, mundos a priori universales y mundos irreduciblemente particulares, y que por lo tanto transforman de manera radical la superficie sobre la que el sentido se constituye y reconstituye una y otra vez, se subvierte y vuelve a emerger, contingente, recortado sobre escenas culturales locales y globales.

Quizá sea importante anotar aquí que lo que me interesa pensar en torno al significante francés no tiene nada que ver con la visión de Pascale Casanova en su La república mundial de las letras, donde plantea la idea de un campo literario modernista organizado alrededor de la función reguladora de París como centro que consagra textos y autores provenientes de todas las esquinas del mundo, reproduciendo tanto las relaciones desiguales que conforman el campo como una noción normativa de valor estético. Y uso aquí la noción de reproducción en un sentido marxista, como la manera en la que la sociedad recrea su orden hegemónico, como la suma de procesos materiales y procedimientos de significación en función de los que se reinstituyen y reafirman las condiciones sociales y epistemológicas sobre las que se funda lo social. Muchos académicos importantes han criticado el hecho de que el mundo de Casanova está estructurado alrededor de circuitos y negociaciones mediadas siempre por el lugar simbólico y hegemónico de París ${ }^{3}$. David Damrosch, por ejemplo, criticó el "triunfalismo implícito" de su perspectiva francesa, y escribió que su libro debería haberse titulado "La République parisienne des lettres". Por supuesto, el problema con Casanova no es que trabaje alrededor de una explicación particularmente francesa del sistema literario mundial, porque todas las descripciones de la literatura mundial son particulares o, mejor, modos particulares de articular imaginarios universalistas, y es imprescindible, siempre, subrayar las determinaciones particulares que constituyen sus presupuestos histórica y culturalmente variables: mundialidades del mundo en sus formulaciones contingentes ${ }^{4}$. El problema (y este creo que es el punto de Damrosch) no es que el mundo de Casanova sea ostensiblemente

\footnotetext{
Christopher Prendergast: "a process whereby historically France, and more particularly, Paris, emerges as the dominant force in the shaping of the Republic of Letters... the center par excellence of the international literary order". But for this critical perspective to be effective "at a deeper level the argument would require a more theoretically robust explanation than that implied by purely anecdotal accumulation" (8).

David Damrosch mismo describe, en What is World Literature?, su literatura mundial en términos perspectivistas y relativos: "For any given observer, even a genuinely global perspective remains a perspective from somewhere, and global patterns of the circulation of world literature take shape in their local manifestations. With this in mind... I will be concentrating particularly (though not exclusively) on world literature as it has been construed
} 
francés, tal como se evidencia en su comprensión extremadamente francesa de la formación liberal del campo literario mundial implicada en el concepto de 'república'; el problema reside en el intento hegemónico de universalizar ese mundo, en presentar la particularidad político-cultural del mundo francés como el principio unificador de la totalidad del sistema literario modernista mundial. Lo que me interesa en este artículo es complicar esta narrativa que lee a los modernistas marginales solo en función de su deseo de París, de sus intentos (reales por otra parte) por ascender al cielo parisino desde las bajas periferias de la modernidad global, y que toman, conquistan y se apropian del capital estético de la modernidad global que los autorizará y que modernizará las literaturas menores de las que provienen, marcando los límites de sus restringidas intervenciones estéticas modernistas:

Aquellos quienes, como Darío, Paz, Kiš, y Benet, van al centro para buscar -para comprender, asimilar, conquistar, robar...- riqueza literaria y posibilidades que hasta ese momento les habían sido negadas, ayudan a acelerar el proceso de construcción de activos literarios en las pequeñas naciones del mundo (326).

Casanova describe, así, una dinámica de reproducción (insisto reproducción como la permanente reinstitución de las condiciones de posibilidad de un orden hegemónico, en este caso, con París en el centro), según la cual el sentido de la intervención de los modernistas marginales es reducido apenas a perpetuar la organización francesa del sistema modernista que los margina y los condena a la enunciación mimética.

De ninguna manera quiero minimizar la importancia del significante francés en la formación de un campo global de producción literaria modernista; lo que me interesa es revisar la noción convencional de cosmopolitismo como la inscripción de una forma geométrica euclidiana de la distancia efectiva que separa objetos marginales deseantes del objeto-causa de su deseo (sea este París, Londres, Nueva York o cualquier otro lugar imaginario de plenitud autoreconciliada), para pensar al cosmopolitismo como una relación de distancias dislocadas: el cosmopolitismo como una manera de desplazar afectivamente tal brecha geométrica, transformando la distancia efectiva en proximidad afectiva; el cosmopolitismo como una dislocación afectiva de la distancia, o más bien, una forma afectiva de distanciarse y trastocar la distancia que desfonda y estría,

over the past century in a specific cultural space, that of the formerly provincial and now metropolitan United States" (27-8). 
pliega y agujerea el espacio de la literatura mundial, tornando imposible o ridícula cualquier idea más o menos sociológica de circulación global.

Lejos de reproducir el lugar hegemónico y estable de París o la distancia estable que la separa de, digamos, América Latina, los cosmopolitas modernistas que me interesan (como Jules Supervielle, sobre el que voy a presentar algunas ideas en la segunda parte de este artículo), la desplazan, dislocan y reconfiguran. La consecuencia más obvia de enfocarse en estas operaciones de dislocación, desplazamiento y reconfiguración de relaciones de distancia hegemónicas, que estructuran mundos realmente existentes, es la emergencia de redefiniciones intersticiales de los discursos críticos de la literatura mundial y el modernismo global que ya no afirmarían (que ya no podrían afirmar) mundos, sino que leerían su crisis, sus condiciones de imposibilidad. Ya no se trata de mundializar (to world como verbo, tal como lo utiliza Pheng Cheah, quien toma la noción de Ser y Tiempo de Heidegger), de hacer mundo, de registrar las maneras en las que literatura moderna, en efecto, creó el mundo; se trata en cambio de leer el fin del mundo (del estable mundo francés de Casanova, por ejemplo), entender que el mundo no existe, que ya no existe como totalidad de sentido y como estructura de la significación crítica y estética, que solo tenemos intersticios sin mundo, ruinas de eso que fue mundo y que ya no es. Y este mundo que ya no es mundo de hoy, este mundo no-mundo de 2017 se puede leer en zonas particularmente perceptivas y atentas de la literatura contemporánea, pero también en algunos textos de cosmopolitas/modernistas intersticiales que lo anticiparon, que supieron ver las grietas de la totalidad simbólica que todavía era mundo. Supervielle pudo figurar ese mundo dislocado, precisamente, por su colocación estético-geopolítica intersticial, porque no pertenecía a ningún lugar de manera plena, y entonces, desde ese lugar intersticial y anacrónico, figuró un mundo dislocado que ilumina nuestro propio no-mundo de hoy, en el que ya no existen los lugares estables separados por distancias geométricas que ordenen el espacio de la diferencia cultural. Y en su mundo dislocado de 1923 entreveo la verdad de 2017: que escribimos y leemos sobre los escombros que quedan de un mundo que ya no es (que no puede ser) horizonte de lo común, porque lo único que compartimos es la universalidad de nuestra extranjería. Los modernistas intersticiales como Supervielle, que no pertenecen de manera plena a ninguna de las culturas sobre cuyas superficies se deslizan, que viven en las grietas de un mundo dislocado, imaginan mundos reconfigurados, reensamblados, mundos como collages aparentemente sin sentido o lógica, marcados de manera indeleble por antagonismos y exclusiones que reduplican su dislocación, mundosmenos-que-mundos, mundos sin mundialidad, y entonces, mundos en los que la marginalidad de sus prácticas estéticas dislocadoras encuentra un lugar, o no, porque en un mundo que es menos que mundo y que ya no puede ser pensado 
como totalidad de sentido no hay lugares propios idénticos a sí mismos, no existen más los privilegios ontológicos, la experiencia de pertenencia es imposible (y su enunciación es puramente ideológica). Las cartografías dislocadas de Supervielle anticipan, entonces, este mundo no-mundo de 2017 en el que la única condición generalizada es la extranjería común que define la vulnerabilidad de los intentos fallidos por habitar sus intersticios y hendiduras.

\section{L'HOMME DE LA PAMPA DE JULES SUPERVIELLE Y LA DISLOCACIÓN EL MUNDO}

En un brillante ensayo sobre la novela radical y experimental L'Homme de la pampa (1923) del escritor franco-uruguayo Jules Supervielle, Sylvia Molloy analiza su escritura intersticial en relación con la imposibilidad de decidir si es que fue "a French poet born in Uruguay or a Uruguayan who became a French poet" (273) [un poeta francés nacido en Uruguay o un uruguayo que se volvió un poeta francés $\left.{ }^{5}\right]$. Supervielle nació en Uruguay en 1884 de padres franceses, y se trasladó a Francia a los diez años; años más tarde se casó con una uruguaya en París y siempre volvió a Uruguay para pasar largas temporadas en el campo de su familia, y mantuvo intensas relaciones intelectuales con escritores y artistas vanguardistas argentinos y uruguayos. Perfectamente bilingüe, era según su propia definición un "poéte des deux rivages" (Molloy 273), lo que subraya su doble pertenencia. Creo que esa afirmación de un surplus de pertenencia es inexacta porque Supervielle no tuvo una inserción cómoda ni en París entre los surrealistas, ni en el Río de la Plata donde era bien recibido pero siempre como un extranjero; por eso me interesa pensarlo a través del concepto de lo intersticial que, por lo menos, posterga cualquier expectativa de pertenencia plena y sugiere la precariedad-no socio-económica pero sí epistemológica-de las condiciones de producción discursiva.

En 1923 publicó en Gallimard L'Homme de la pampa, traducido al español en 1925 por el poeta vanguardista Juan Parra del Riego, aunque Supervielle tuvo la posibilidad de intervenir y editar la traducción. Supervielle no integró ningún grupo surrealista y tuvo una relación típicamente conflictiva con André Breton, no obstante, Keith Aspley explica que, a partir de la década de 1950, la 
tradición literaria leyó buena parte de su producción narrativa y poética como una contribución a la poética surrealista (15). Y, sin embargo, L'Homme de la pampa es una novela que juega con el sinsentido, inventiva y divertida, con una trama absurda que no apuesta a ningún tipo de coherencia narrativa, y descarta cualquier noción de verosimilitud.

Aunque las nociones de novela y personaje no sirvan para pensar este texto que, en la tradición de la vanguardia histórica constituye su estética en el intento de destruir la institucionalidad del arte y las categorías que estabilizan la especificidad genérica de la literatura, voy a dar cuenta del delgado argumento para concentrarme en las operaciones que encuentro más relevantes en el texto, en relación con la dislocación radical del mundo que despliega Supervielle. Entonces: el dispositivo vaciado de sustancia histórica que no-es-personaje de esta no-novela, Juan Fernández y Guanamirú, es un uruguayo rico viviendo en el campo con treinta hijos ilegítimos, uno de los cuales es el "contremaître" o capataz de su estancia ${ }^{6}$. Fernández y Guanamirú es víctima de un aburrimiento mortal ("le mal du désert dont il avait eu longtemps à souffrir alors qu'il vivait à l'estancia. L'affection [a été] provoquée par une immense oisiveté dans la campagne sans limites" (35) [la enfermedad del desierto que él había sufrido mientras vivía en la estancia. La afección \{fue\} provocada por un inmenso aburrimiento en el campo sin límites]) y decide construir un palacio gigante. Pero el palacio no logra interrumpir su terrible aburrimiento, e inspirado por un libro que encuentra en su biblioteca, se le ocurre la idea de construir un volcán: "un volcan, mon ami, un volcan qui honrera le pays" (43) [un volcán, mi amigo, un volcán que honrará al país]. Investiga sobre diferentes volcanes de Japón, El Salvador, Nicaragua, China, Ecuador e Italia, y termina escogiendo un volcán propio, un modelo nuevo, "un volcan jeune" (46) que será la síntesis de los volcanes mundiales, y lo llama "Future". Después de un año de trabajo, acaba su volcán y lo considera una obra de arte: "Je suis un artiste!" (50) [iSoy un artista!], and "quoi de plus beau qu'un volcan, cette reaction de la matière fluide et ignée contra croûte terrestre consolidée?" (51) [¿Qué hay más bello que un volcán, esa reacción de la materia fluida e ígnea contra la corteza terrestre consolidada?]); el volcán es una obra de arte vanguardista que desestabiliza las ideas convencionales de belleza; o más bien, un trabajo experimental de no-arte

$6 \quad$ Supervielle dio a Juan Fernández y Guanamirú un doble apellido, indicando su estatus social (la mayoría de las familias patricias del Río de la Plata utilizan dos apellidos), pero en este caso, uno es español y el otro de origen guaraní, dislocando y descentrando su posición socio-económica mediante una identidad híbrida. 
como la institución burguesa cuya crisis la novela tematiza $y$ performa ${ }^{7}$. Pero incluso después de haber logrado esta proeza, su aburrimiento no se extingue, y entonces decide viajar a París a presentar su invención: "Emporter le volcan, mais oui, puisqu'on était indigne de le comprendre dans ce pays" (64) [Llevarse el volcán, sí, porque no somos dignos de comprenderlo en este país]. Fernández y Guanamirú le encarga a un equipo de trabajadores rurales, bajo la supervisión de su hijo-capataz, la tarea de desarmar el volcán, cortarlo en numerosas rebanadas, y empacarlo en containers para el viaje transatlántico a Francia, pero los peones rurales hacen muy mal su trabajo y arruinan el volcán: "De sa montagne ardente il ne reste plus, intacte, que la housse imperméable dont on la recouvrait les jours de pluie" (70) [De esta montaña ardiente no queda más que, intacta, la funda impermeable que utilizaba en los días de lluvia]. En el contexto de una narrativa simbolista, la pérdida del volcán llamado "Future" abriría el espacio interpretativo a lecturas alegóricas sobre la crisis eminentemente moderna del horizonte de futuridad y de una relación histórica teleológica, pero en L'Homme de la Pampa la destrucción de Future es apenas una peripecia vaciada de historicidad: a bordo del transatlántico que lo va a llevar a Europa, Juan Fernández y Guanamirú descubre una réplica miniatura del volcán arruinado que, no está claro cómo, aparece entre su ropa, en su valija. Cuando llega a Burdeos y luego a París, la estructura narrativa del texto cambia: si la sección uruguaya de la trama podría caracterizarse como un costumbrismo de nonsense rural, una vez en Francia la no-novela se transforma en una fantasía onírica. París se asemeja a un set de filmación, y los paisajes urbanos parecen reproducciones de postales turísticas. Fernández y Guanamirú se enamora de una mujer bizarra, Line du Petit Jour, que siempre está vestida con un tailleur y que lleva una cartera con la forma de la isla de Córcega, y que aparece y reaparece en momentos sin que la trama justifique sus ausencias y presencias. Sobre el final, Line du Petit Jour lo abandona, también el volcán lo deja, y Fernández y Guanamirú explota en una erupción gigantesca de lava que proyecta imágenes de aves, plantas y ganado

7 Oscilo entre considerar el volcán una obra vanguardista y una obra experimental para subrayar la tensión en la novela que apunta a leerla en diálogo con la vanguardia histórica $y$, a su vez, como una parodia de la auto-representación histórica de la vanguardia. Por otra parte, Lucas Mertehikian me sugirió la posibilidad de leer la construcción del volcán como figura privilegiada de la naturaleza americana, sobre todo en relación con las crónicas de viaje y ensayos naturalistas de Alexander von Humboldt, que señala al volcán Chimborazo en Ecuador. En su Essai sur la géographie des plantes (1805), en el que incluye un croquis fascinante del mundo natural con el Chimborazo como epicentro, se puede leer la figura del volcán (capturada por el aparato discursivo del naturalismo científico) como centro del cosmos. Quiero agradecer mucho a Lucas esta sugerente lectura intertextual. 
sudamericano sobre las calles de París. El texto explica que su muerte se debió a un "éclatement de mégalomanie éruptive, parmi de nuages de cendres, de soufre volcanique et une horrible lave" (219) [estallido de megalomanía eruptiva, en medio de nubes de ceniza, azufre volcánico, y horrible lava].

Esta glosa incompleta del alocado argumento deja en claro que L'Homme de la pampa es una novela sobre viajes, desplazamiento y dislocación, y que Fernández y Guanamirú está siempre en movimiento: desde la primera línea que lo sitúa en un tren "qui l'emportait vers le Nord, tête nue à la portière, il laissait le vent champètre jouer sur son crâne" (11) [que lo llevaba al Norte, la cabeza desnuda por la puerta, dejó que el viento del campo jugara con su cráneo], hasta el último párrafo, cuando el capitán de un barco navegando a través del Pacífico cerca de Borneo ve las manos de Fernández y Guanamirú flotando en el aire, saludándose, después de la explosión de su lava interior que lo despedazó y mandó sus miembros volando a diferentes partes de ese mundo también fragmentado y re-ensamblado: "[ces mains] étaient celles de Guanamirú qui se retrouvaient après un bon voyage en sens opposé, tout autour de la terre" (220) [(esas manos) eran las de Guanamirú, que se encontraban después de un buen viaje alrededor del mundo en sentido opuesto $]^{8}$. Y es en este sentido que me interesa un pasaje de las desventuras de este uruguayo en París, cuando conoce a la mujer de la que se enamora. Line du Petit Jour le cuenta que acaba de regresar "d'un double voyage autour du monde, de l'Est à l'Ouest dans les bras d'un poète haïtien, puis, en sens inverse sur le genoux d'un peintre scandinave" (142) [de un doble viaje alrededor del mundo, de Este a Oeste en los brazos de un poeta haitiano, y después, en sentido inverso, sobre las rodillas de un pintor escandinavo]. Y continúa:

Si je pouvais de moins brouiller des pays comme des dominos!... Pousser un peu la Patagonie vers le Nord, le Groënland vers l'Est, donner aux pôles une allée de palmistes. Est-il admissible qu'après mille siècles d'adorable persévérance les fleuves prennent tous leur source et se

\footnotetext{
Uno de los pasajes más significativos en la serie de tematizaciones de viaje y desplazamiento es cuando Fernández y Guanamirú se topa con un signo que parece ser real solo para desaparecer cuando él lee: "EMPORTER LE VOLCAN EN EUROPE" [Traer el volcán a Europa]. Comienza, entonces, a cuestionar el sentido de la palabra "emporter" ("Que signifiait d'abord le mot emporter? S'il découvrait le sens exact..." (64) [Pero ¿qué significa primero la palabra 'llevar'? Si el descubriese el sentido exacto...]), encuentra un diccionario Larousse y comienza a buscar sinónimos, como en un desplazamiento lacaniano, metonímico, de significantes, una vez más tematizando el problema de nombrar un significado perpetuamente en movimiento (65).
} 
jettent dans la mer exactement au même point et que change seule l'eau qui les forme? Composer de nouveaux paysages! (144-5) [i $\mathrm{Si}$ pudiera al menos barajar países como piezas de dominó!... Poner un poco de la Patagonia hacia el Norte, Groenlandia al Oeste, y regalar a los polos una avenida de Palmeras. Es admisible que después de mil siglos de adorable preservación los ríos todos tomen su fuente y se arrojen al mar exactamente al mismo punto y que cambie solo el agua que los contiene. ¡Ay, componer nuevos paisajes!].

Fernández y Guanamirú confiesa nunca haberse subido al Métro y Line du Petit Jour lo invita a viajar con ella. Lo toman en la estación Hôtel de Ville, pero justo después de pasar por la parada del Palais Royal, ella le pide que se fije dónde están. Él dice que no puede ver nada, a lo que Line du Petit contesta: "En réalité, mon bon ami, nous sommes à la frontière mexicaine et je vous le prouverai à la première occasion... Ne voyez-vous pas que le chef de gare, quoi qu'il fasse, est un indien tout cru, que ses yeux ne sont pas français et qu'il a l'air contrarié des aztèques?" (150) [En realidad, mi buen amigo, estamos en la frontera mexicana y yo se lo probaré a la primera ocasión que tenga. ... ¿No ve que el jefe de estación, cualquiera sea la cosa que haga, es totalmente un indio, que sus ojos no son franceses y que tiene el aire contrariado de los aztecas?]. El uruguayo propone que deberían viajar en el Metro todo el camino hasta Japón, y ella responde: “Le Japon ce n'est pas facile, il faudrait changer trente-deux fois de train et traverser douze fois la Seine. La compagnie ne peut donner aucune indication sur l'heure d'arrivée et il y aurait vraiment trop de gares" (152) [Ir a Japón no es fácil, tendríamos que cambiar de tren treinta y dos veces y cruzar doce veces el Sena. La compañía no puede dar indicación alguna sobre la hora de llegada y habrá allí de seguro muchas estaciones]. Después de cruzar la frontera mexicana para visitar San Diego y pasar por una sala de cine en la que está tocando una orquesta sinfónica, ven la estación de Métro de Couronnes, y "la jeune femme et Guanamirú remontèrent enfin au rez-de-chaussée parisien" (153) [la joven y Guanamirú volvieron a subir al nivel de las calles parisinas].

Estas escenas de dislocación geográfica que, al mismo tiempo, universalizan y dislocan el lugar de París en la escritura cartográfica de Supervielle me resultan fascinantes. Si la dislocación es el desajuste, descentramiento y degradación de una estructura que enfatiza su no-coincidencia consigo misma, Line du Petit du Jour, como el personaje que encarna el quiebre del orden de significación que alude al internacionalismo y a la naturaleza radical de la vanguardia, funciona como la figuración de la agencia dislocadora del texto que interviene formaciones 
geográficas y baraja países como fichas de dominó. El mundo no-mundo de L'homme de la Pampa está hecho de contigüidades imposibles mediadas por el subterráneo parisino, según las que México, San Diego y Japón funcionan como dislocaciones internas de un París que no es (no puede ser, no puede seguir siendo) idéntico a sí mismo; México, San Diego y Japón son figuras de un otro dentro de París que lo fractura y lo vuelve irreconocible, desplazándolo del centro del sistema de significación geomodernista, que aparece ahora completamente desestructurado9.

Si L'homme de la Pampa fuera una Bildungsroman, no importa qué novela de formación querramos invocar, las desventuras del viaje ascendente de las periferias hacia París culminarían con un homecoming reconciliado que reafirmaría una relación dialéctica estática entre lo universal y lo particular en función de una identificación apaciguadora que pudiera preservar la diferencia ontológica entre el lugar de la hegemonía que encarna el significante francés y sus diferentes

$9 \quad$ Es interesante comparar la dislocación cartográfica en juego en la novela de Supervielle con una reconfiguración geográfica contemporánea del mundo que tiene afinidades y discontinuidades con L'Homme de la pampa: "Le Monde au Temps Surrealistes" (1929) [El mundo en tiempos del surrealismo], un mapa global dibujado a mano, publicado en el periódico belga Variétés sin citar autor alguno, aunque se ha presupuesto durante largo tiempo que fue producido por el poeta dadaísta y surrealista Paul Éluard. En una versión más larga de este ensayo exploro esta comparación más profundamente, pero es suficiente destacar aquí que la táctica de Supervielle es marcadamente más radical que el descentramiento en el mapa surrealista de océanos (particularmente la nueva centralidad del Pacífico), continentes y regiones (en específico, el gigantismo de Alaska, Labrador, Tierra del Fuego, México, Isla de Pascua y Rusia, en oposición a la miniaturización de Europa, Estados Unidos, África y Sudamérica).

En relación con esta comparación, quisiera ofrecer una última interrogante sobre si acaso la novela de Supervielle reproduce o disloca la función estructuradora de París en el contexto del modernismo global de París o llanamente la socava. Esta es una pregunta justa que surgió al presentar estas ideas en mi conferencia en el Institute for World Literature en julio del 2016. Alguien de la audiencia preguntó: ¿Por qué el "retorno" de la novela a París como su escenario privilegiado es una forma de cosmopolitismo negativo que disloca el mundo, y no una reproducción de la ideología francófila? Mi respuesta fue: creo que el único modo en el que se puede dislocar un mundo, entendido como una totalidad internamente estructurada de sentido, es apuntando a la instancia mediadora efectiva que asegura (o solía asegurar) la formación del mundo como totalidad modernista. En otras palabras, la maniobra dislocadora de Supervielle es realmente efectiva porque precisamente se enfoca en París. No podría imaginar que esta novela hubiese sido casi tan eficaz si hubiese escogido como escenario a Montevideo, El Cairo, Sidney o Shanghai como el sitio y dispositivo de su deshacimiento del mundo o des-mundanización. 
márgenes geopolíticos ${ }^{10}$. Pero la de Supervielle no es una Bildungsroman, y ni siquiera es una novela; sus dislocaciones del mundo producen una cartografía sin distancias estables o mensurables, sin posiciones fijas o predecibles que socava la estabilidad necesaria para las mediaciones hegemónicas que son necesarias para la constitución del discurso francófilo, y sirve como un antídoto contra la fetichización de la diferencia cultural que permea muchos discursos críticos postcoloniales y subalternistas.

La literatura mundial y el modernismo global intersticiales alrededor de los que me interesa pensar suponen intervenciones estéticas y críticas que crean cartografías afectivas, dislocadas, cuya función no es la de postular mundos alternativos, mundos felices, sino la de mostrar las fracturas y las crisis de esto que ya no es mundo; en el que no hay lugares, solo permanentes desplazamientos sinsentido dentro de un París que es México, y es San Diego y es Japón, aunque México, San Diego y Japón no sean México, San Diego y Japón sino los nombres catacréticos de una fuerza dislocadora interior a París que le impide volverse mundo, esto es, que impide que estructure un mundo modernista a su alrededor a imagen y semejanza del significante francés. La lógica demencial de L'Homme de la Pampa provoca la implosión del mundo y lo entierra bajo "nubes de ceniza, azufre volcánico, y horrible lava", pero no instituye un nuevo mundo, un nuevo volcán, un nuevo Future, una nueva figura de la totalidad planetaria, no, nada; y esto nos devuelve al comienzo: el mundo no existe.

\section{BIBLIOGRAFÍA}

Aspley, Keith. Historical Dictionary of Surrealism. Lanham, Toronto and Plymouth: The Scarecrow Press, 2010.

Casanova, Pascale. La república mundial de las letras. Barcelona: Anagrama, 2001.

Cheah, Pheng. What Is a World?: On Postcolonial Literature as World Literature. Durham: Duke University Press, 2016.

Damrosch, David. What Is World Literature?. Princeton: Princeton University Press, 2003.

Goethe, Johann Wolfgang von. Conversations with Eckermann: Being Appreciations and Criticisms on many Subjects. New York: M.W. Dunne, 1901.

Humboldt, Alexander von. Essai sur la géographie des plantes : accompagné d'un tableau physique des régions équinoxiales, depuis le dixième degré de latitude boréale jusqu'au

10 Sylvia Molloy, por ejemplo, compara el texto de Supervielle con Raucho y Don Segundo Sombra, ambas de Ricardo Güiraldes. 
dixième degré de latitude australe, pendant les années 1799, 1800, 1801, 1802 et 1803. Paris: Levraut, Schoell et Compagnie, 1805.

Laclau, Ernesto y Chantal Mouffe. Hegemonía y estrategia socialista. Hacia una radicalización de la democracia. Madrid: Siglo XXI, 1987.

Molloy, Sylvia. "Traffic in translation: Rereading Supervielle". French Global. A New Approach to Literary History. Ed. Christie McDonald y Susan Suleiman. Nueva York: Columbia University Press, 2011.

Prendergast, Christopher. "TheWorldRepublic of Letters". Debating World Literature, Christopher Prendergast, ed. New York and London: Verso, 2004, 1-25.

Supervielle, Jules. L'Homme de la Pampa. Paris: Gallimard, 1923. El hombre de la pampa. Montevideo: Arca, 1969. 